\title{
Phase coherent transport in two coupled quantum dots
}

\author{
R.H. Blick ${ }^{a}, *$ A.K. Hüttel ${ }^{a}$, A.W. Holleitner ${ }^{a}$, E.M. Höhberger ${ }^{a}$, H. Qin ${ }^{a}$, \\ J. Kirschbaum ${ }^{\text {a }}$,J. Weber ${ }^{\mathrm{a}}$, W. Wegscheider ${ }^{\mathrm{b}}$, M. Bichler ${ }^{\mathrm{c}}$, K. Eberl ${ }^{\text {d }}$, J.P. Kotthaus ${ }^{\mathrm{a}}$ \\ ${ }^{a}$ Center for Nanoscience and Sektion Physik, Ludwig-Maximilians-Universität, \\ Geschwister-Scholl-Platz 1, D-80539 München, Germany \\ ${ }^{\mathrm{b}}$ Institut für Angewandte und Experimentelle Physik, Universität Regensburg, D-93040 Regensburg, Germany \\ ${ }^{\mathrm{c}}$ Walter-Schottky-Institut, Technische Universität München, D-85747 Garching, Germany \\ d Max-Planck-Institut für Festkörperforschung, Heisenbergstr. 1, 70569 Stuttgart, Germany
}

\begin{abstract}
Quantum dots by now offer a well-defined environment for studying quantum physics. Hence, various proposals have been introduced how to integrate these artificial molecules for building quantum computing devices. Crucial for operating such circuits is the realization of wave function coherence established in coupled quantum dots. Consequently, the foremost goal is to devise basic circuits for testing phase coherence and dissipation mechanisms.
\end{abstract}

PACS: $73.63 . \mathrm{Kv} ; 03.65 .-\mathrm{n} ; 73.40 . \mathrm{Gk}$

Keywords: Quantum dots; Quantum mechanics; Tunneling

Current work on quantum dots laterally embedded in two-dimensional electron gases is focussing on the close analogy of coupled dots to real molecules. Various proposals have been introduced how to integrate these artificial molecules for building quantum computing devices. Such a quantum computer should be capable of solving specific tasks classical computing machines are not powerful enough for. While the main promise for quantum computing schemes based on dots is the scalability of semiconductor computing elements, yet the very basic circuits and operations with these specific quantum bits (qubits) have to be devised and tested. The beauty of applying quantum

\footnotetext{
* Corresponding author. Tel.: +49-89-2180-3733; fax: +49-892180-3182.

E-mail address: robert.blick@physik.uni-muenchen.de (R.H. Blick).
}

dots as quantum computational elements is the flexibility these systems offer as compared to real atoms. The avenue we are taking in the course of this work is to study two coupled quantum dots forming a molecular mode which we treat as a qubit. This enables to fully control and engineer the interaction of a qubit with the environment (dephasing) and with other qubits (communication). Dephasing is introduced via the electron-electron interaction, electron-spin and electron-phonon coupling. Naturally, the electronelectron and electron-photon interactions have to be considered as means for communicating, i.e. addressing, the individual qubits. Another quite interesting aspect is the spin coupling of electrons and the nuclei of the semiconductor structure in which the quantum dots are embedded.

The basis of all experimental approaches to quantum computation is the definition of a proper qubit: 
For quantum dot systems the qubit of choice is the coherent mode generated by discrete states in two quantum dots. The energy scales involved in this setup are the Coulomb interaction $E_{\mathrm{C}}$ of $N$ electrons captured in a quantum dot, the electron spin interaction $E_{\mathrm{S}}$, excited states $\varepsilon^{*}$ of the $N$-electron system, and the tunnel coupled molecular or coherent state $\varepsilon_{\mathrm{t}}$. In our experiments the foremost goal is to achieve large Coulomb energies $E_{\mathrm{C}} \sim 1-3 \mathrm{meV}$ in order to establish a sufficient signal/noise ratio. Connected to this energy scale are the excited states of the $N$-electron systems: Empirically it is found that these scale according to $\varepsilon^{*} \sim E_{\mathrm{C}} / 5-E_{\mathrm{C}} / 3$. This finally enables observation of tunnel coupled states $\varepsilon_{\mathrm{t}}$ of the order of $50-200 \mu \mathrm{eV}$. The advantage of using discrete states of two quantum dots instead of ground and excited states of the same artificial atom lies in the enhanced flexibility of gating two individual dots. At the same time variation of the coupling strength easily allows for activating or deactivating of single qubits.

The material system of choice are $\mathrm{AlGaAs} / \mathrm{GaAs}-$ heterostructures containing a high-mobility twodimensional electron gas with a phase coherence length of typically $10-30 \mu \mathrm{m}$ at low temperatures. Definition of quantum dots is usually achieved by electron beam lithography and adjacent deposition of Schottky field-effect gates. Quantum dots contain roughly $10-100$ electrons and are attached to metallic leads by tunneling barriers. The main advantage of heterostructures is the high degree of perfection with which its electronic, photonic and phononic properties can be tailored. These materials already enabled ground breaking work which demonstrated that quantum dots in the few electron limit show not only charge quantization, but reveal a discrete energy spectrum similar to real atoms [1]. While it might not be possible with laterally integrated quantum dots to build a full scale quantum computer, these dot circuits are the ideal model systems for studying the principles of operation, which later on might be implemented in molecular devices.

In this work we focus on a four-dot device as shown in Fig. 1, featuring a setup for sampling the interaction of two qubits. The single qubit in this approach is formed by a superposition of two discrete states in two coupled dots. Individual source and drain contacts allow simultaneous addressing of both qubits. Several gating electrodes enable to directly switch the entan-

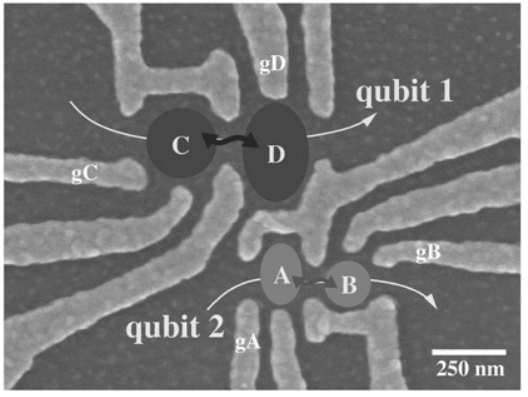

Fig. 1. Quantum dot circuit with four dots forming two quantum bits - the qubits A-B and C-D are coupled capacitively in this case.

glement in the qubit circuit. The qubits considered in this context are defined on the basis of electronic charge, i.e. on the spatial component of the wave functions. Although the electron's spin on the other hand can be manipulated in certain cases [2], spin resonance detection for single quantum dots is still in its infancy [3].

Of prime interest here is entanglement of a qubit and back-action of the probing mechanism on the phase coherent quantum state. Entangling qubits will allow to introduce XOR-operations and hopefully error correction mechanisms. As introduced above dephasing of coherent electronic modes via coupling to the phonon bath has to be considered. After introducing measurements on tunnel coupled quantum dots, i.e. a single qubit, a phase sensitive detection scheme for electronic wave functions is introduced [4]. This scheme is easily expanded for non-invasive probing truly molecular states in coupled dots [5]. Finally, a fabrication technique for high-mobility low-dimensional electron systems in phonon cavities is presented and first measurements are discussed [6]. We will not focus on the electron-photon interaction, however, it should be noted that we succeeded recently to probe electronic coherent modes in a qubit by microwave radiation in the frequency range of $1-40 \mathrm{GHz}$ [7]. This gives sampling rates of $1-50 \mathrm{~ns}$ with which qubits with life times of $1-100 \mathrm{~ns}$ can be addressed.

The devices we use are realized in a two-dimensional electron gas (2DEG) being $90 \mathrm{~nm}$ below the surface of an $\mathrm{AlGaAs} / \mathrm{GaAs}$ heterostructure. At a bath temperature of some $10 \mathrm{mK}$ the electron mobility and the 

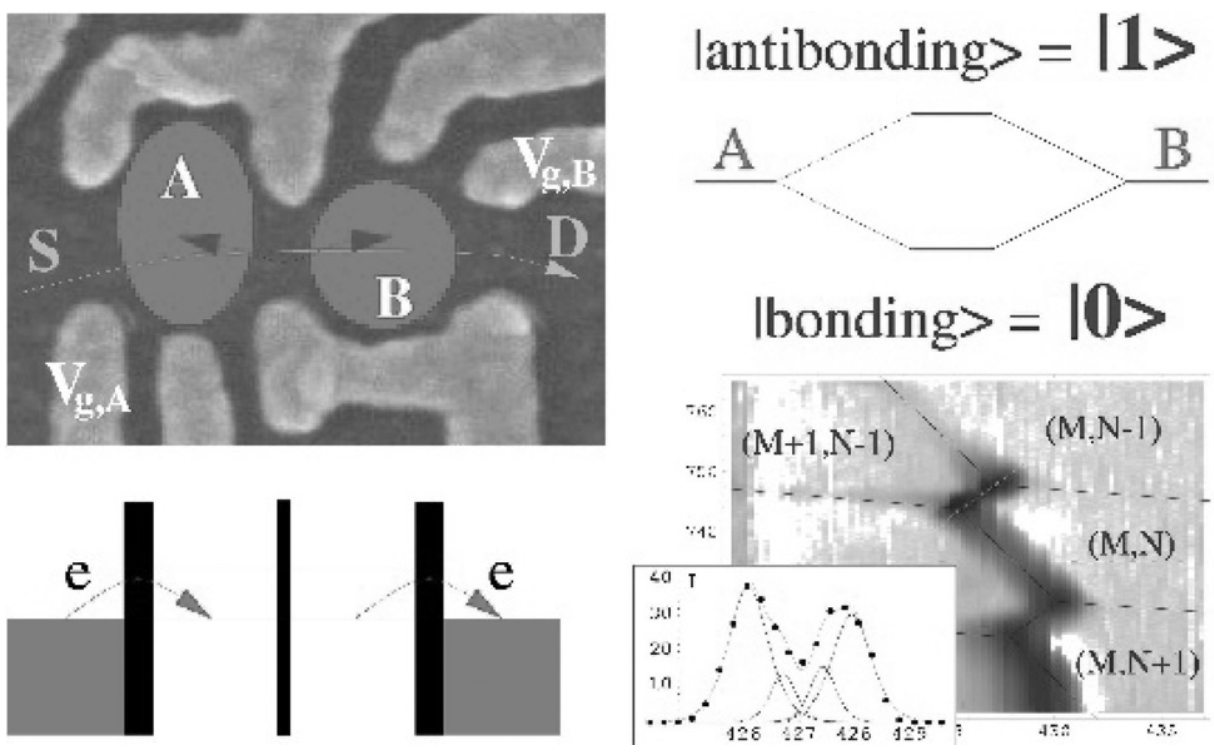

Fig. 2. A single qubit A-B is tuned into resonance with the center gate tuning the overlap of the wave functions in A and B. For a strong overlap the tunnel splitting leads to the formation of a bonding and an anti-bonding state. In the lower right half of the figure a charging diagram of the two dot system is shown. The charge states are indicated by the number of $(M, N)$ electrons in dot $\mathrm{A}$ and $\mathrm{B}$. The dark lines represent a non-zero current through the dots, intersecting the different charging states. The inset is a single data trace taken from the main diagram, obviously not only two ground (binding) states of the $(M+1, N)$ and $(M, N-1)$ contribute to the electronic transport, but additionally the two anti-binding states are involved. The tunnel splitting in this case is of the order of $50-70 \mu \mathrm{eV}$.

density are usually found to be $\mu=8-12 \times 10^{5} \mathrm{~m}^{2} / \mathrm{V} \mathrm{s}$ and $n_{\mathrm{s}}=1-3 \times 10^{15} / \mathrm{m}^{2}$, respectively. By electron beam writing and Au-evaporation Schottky-gates are defined. Under appropriate voltage bias these form the desired quantum dots. As seen in the scanning electron microscope micrograph of Figs. 1-3, the gates also define the tunneling barriers between the two quantum dots. By pinching off the tunneling barriers of one of the dots, we first characterize each dot individually. From transport spectroscopy we find the following charging energies for the dots to be of the order of $E_{\mathrm{C}}=\mathrm{e}^{2} / 2 C_{\Sigma}=1-2 \mathrm{meV}$. Taking into account the electron density the number of electrons in the dots is estimated to be $10-50$ depending on the dot radii. For the actual measurements the mesoscopic system can be tuned into a regime $2 E_{\mathrm{C}}=U>\varepsilon_{\mathrm{dots}}^{*} \sim$ $\Gamma>k_{\mathrm{B}} T_{\mathrm{e}}$ in which charge transport is dominated by tunneling through single particle levels.

In Fig. 2 a single qubit is depicted with the two individual dots marked as $\mathrm{A}$ and $\mathrm{B}$. When coupled properly through the tunneling barrier, a splitting of the two discrete states in $\mathrm{A}$ and $\mathrm{B}$ occurs into a bond- ing and an anti-bonding state, as shown in the upper right-hand side. The bonding state was assumed to have a lower energy in this case what, however, depends on the spin states of the electrons involved. Such a qubit is then probed by determination of the charging diagram of the system: the gate voltages indicated alter the number of electrons in the two dots, e.g. from $(M, N)$ to $(M+1, N)$, or $(M, N-1)$, etc., see lower right-hand side in Fig. 2. Each time the number of electrons is changed a conductance resonance appears, which is identified by the dark regions in the gray scale plot. Depending on the absolute number of electrons confined, the tunnel split resonance appears as an additional conductance channel for electrons.

In the inset of the charging diagram in Fig. 2 a single line plot from the diagram is taken, crossing the charge states $(M, N-1)$ and $(M+1, N)$. As seen we find a resonance trace which comprises not only the two ground states, but in addition two peaks hidden in the shoulder of the main resonance. These additional resonances are identified as the anti-bonding states of the $(M, N-1)$ and $(M+1, N)$. The energy splitting 


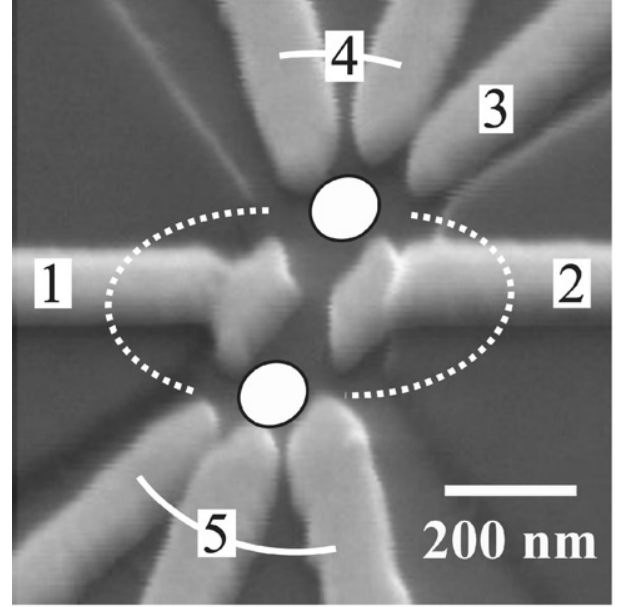

(a)

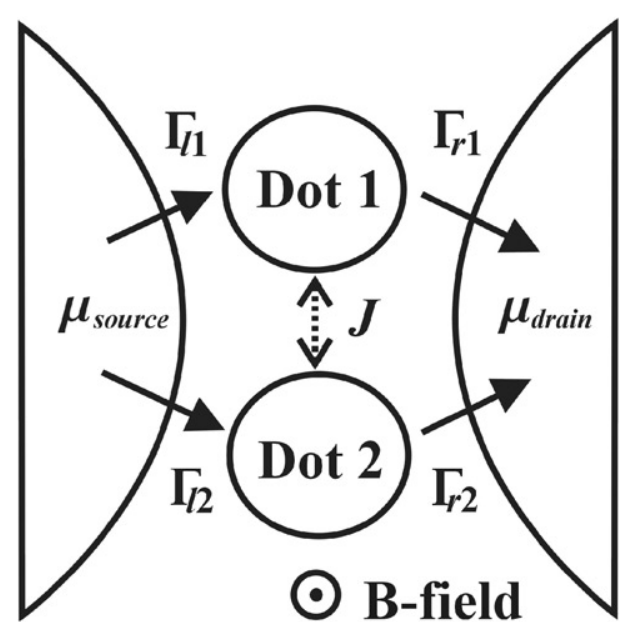

(b)

Fig. 3. (a) Two quantum dots are integrated in the arms of an $\mathrm{AB}$ ring for sampling phase coherence of electronic wave functions in artificial molecules. The device is defined by electron-beam writing in a two step process. (b) By applying appropriate negative voltages to the gates, a two-path quantum dot system is realized. An electron in the source contact can tunnel via both dots into the drain contact. Furthermore the coupling between the two dots can be tuned by voltages.

is of the order of $\varepsilon_{\mathrm{t}} \sim 50-70 \mu \mathrm{eV}$. Evidently, such a coherent mode can be monitored in a coupled dot system. Below we will see how to increase the coupling in order to achieve separate peaks for the bonding states.

In Fig. 3 another setup with two coupled dots is shown, which allows us to probe phase coherence of a single qubit. The two dots are located in the arms of an Aharonov-Bohm (AB) interferometer with tunable source and drain contacts [4]. The particular advantage of this setup is the ability to not only sample the electronic phase, but in addition to vary the overlap of the two discrete states in the dots. This is depicted schematically in Fig. 3(b): drain and source couple to $\operatorname{dot} 1$ and dot 2 via $\Gamma_{l_{i}, r_{i}}$, while the interdot overlap is given by $J$. The magnetic field is oriented perpendicular to the plane of the sample. The measurements on this sample geometry are shown in Fig. 4. In (b) the current through the $\mathrm{AB}$ ring is shown for the weak coupling regime. Ramping now a perpendicular magnetic field, we detect AB-oscillations with a periodicity of $\Delta B \approx 16.4 \mathrm{mT}$. This corresponds to an embedded area of $A=2.52 \times 10^{-13} \mathrm{~m}^{2}$ in good accordance with the lithographic size of the two-path dot system. The two insets in Fig. 4(b) give the Fourier transformation of the oscillations, while the solid triangles indicate phase locking $120 \mathrm{mT}$. The tunnel coupling for the geometry shown in Fig. 3 is represented in Fig. 4(a): Since the dots in this case only contain 15 electrons each, we find a clear splitting marked by the bold line. The coupling energy is found to be of the order of $120 \mu \mathrm{eV}$-as before two resonances are shown. In terms of capacitances we obtain coupling strengths of about $C_{12} / C_{\Sigma}^{\text {dot } 1} \cong$ $C_{12} / C_{\Sigma}^{\text {dot } 2}=0.37 \pm 0.08$.

In order to address the physics of dissipation in the ultimate limit of single electrons interacting with phonon modes of their semiconductor host crystal, the most promising approach is to machine freely suspended nanobridges from structured semiconductor materials. The importance of the electron-phonon interaction in nanostructures was demonstrated by Fujisawa et al. [8] and Qin et al. [7] for non-suspended quantum dots in $\mathrm{AlGaAs} / \mathrm{GaAs}$-heterostructures and theoretically modelled by Brandes and Kramer [9]. Blick et al. [10] recently presented first transport measurements on such 2DEGs and suspended quantum dots.

Free-standing nanostructures were fabricated from MBE-grown GaAs/AlGaAs-heterostructures containing an additional $400 \mathrm{~nm} \mathrm{Al}_{0.8} \mathrm{Ga}_{0.2} \mathrm{As}$ sacrificial layer. The succeeding active layer has a total thickness of $130 \mathrm{~nm}$. It contains a high-mobility 

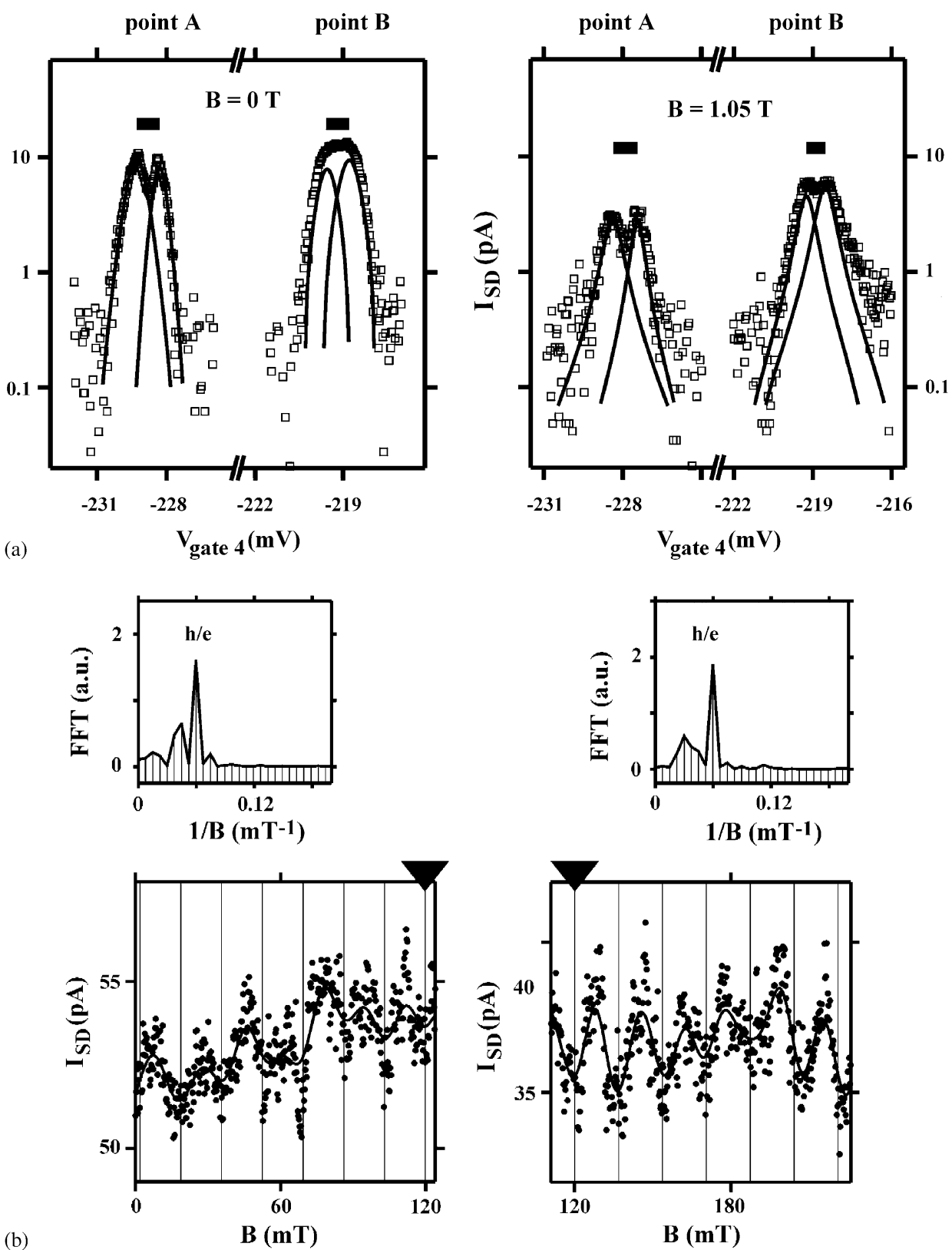

Fig. 4. (a) In the regime of strong coupling we find clear tunnel splitting with about 15 electrons in each dot. (b) In case of weak coupling we operate the device as an $\mathrm{AB}$ interferometer. If a magnetic field is applied perpendicular to the quantum dots, the amplitude of the current produces AB-oscillations as shown. The insets give the Fourier transformation of the oscillations.

two-dimensional electron gas (2DEG) situated $40 \mathrm{~nm}$ below the sample surface which is surrounded by spacer, donor and cap layers on both sides. Processing three-dimensional nanostructures involves a series of both optical and electron beam lithography steps followed by several pattern transfer steps. Suspended quantum dot structures (see Fig. 5(a)) are patterned using electron beam lithography. The sample 


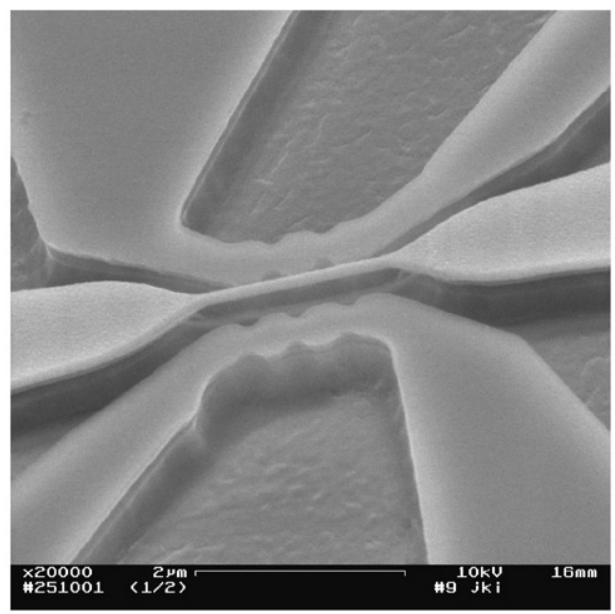

(a)

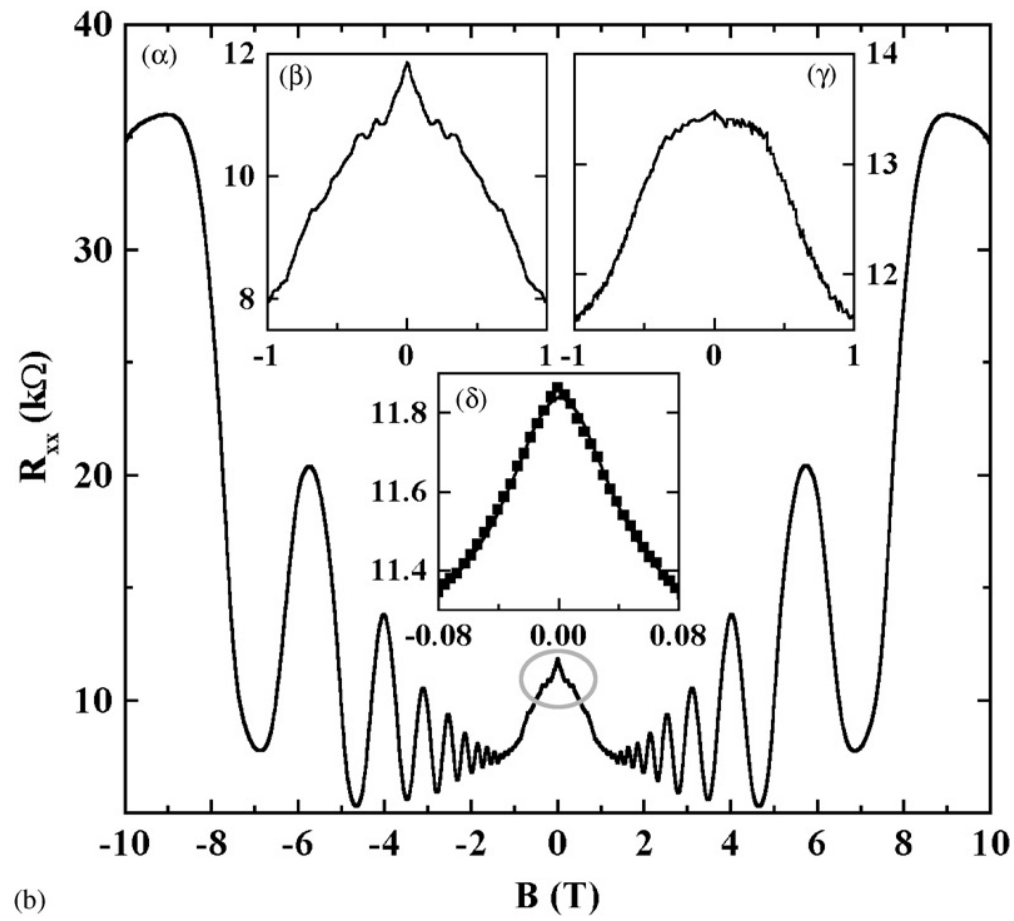

Fig. 5. (a) Top view of a suspended hallbar and quantum dot structure. (b) Shubnikov-de Haas oscillations of a freely suspended quantum cavity measured at $T=5 \mathrm{mK}$ in the high-field domain $(\alpha)$. The insets $\beta$ and $\gamma$ compare the low-field transport properties of a suspended and non-suspended cavity, respectively. In case $\delta$ the weak localization peak of the suspended sample $(\beta)$ is magnified for clarity.

geometry is transferred into the heterostructure by reactive ion etching (RIE) using silane gas. In order to finally suspend the nanostructure, the sacrificial layer is removed by wet etching.
The electronic properties of the 2DEG can be investigated in standard low temperature four-terminal magneto-transport experiments. Shubnikov-de Haas oscillations in the longitudinal resistance of a freely 
suspended hallbar at $T=5 \mathrm{mK}$ are depicted in Fig. 5(b). Via the standard relation $\Delta(1 / B)=g_{\mathrm{s}} e / h n_{\mathrm{s}}$, with spin degeneracy factor $g_{\mathrm{s}}=2$, the carrier density was determined to $n_{\mathrm{s}}=3.19 \times 10^{11} / \mathrm{cm}^{2}$. In addition, at magnetic fields $B>5 \mathrm{~T}$ we find evidence for spin splitting at this temperature. The conductivity at zero magnetic field $\rho_{0}=1 / e \mu n_{\mathrm{S}}$ results in a mobility of $\mu=5.75 \times 10^{4} \mathrm{~cm}^{2} / \mathrm{V} \mathrm{s}$. The obtained properties of the 2DEG imply an improvement compared to previous work [10].

Further information about the electron system can be extracted from additional structure in the longitudinal low-field magnetoresistance. The observed maximum in the low-field region shown in Fig. 5 can be explained as a geometrical resonance of the classical cyclotron radius $R_{\mathrm{c}}=\hbar k_{\mathrm{F}} / e B_{\max }$, with $k_{\mathrm{F}}=\sqrt{2 \pi n_{\mathrm{s}}}$ : The maximum occurs, if $R_{\mathrm{c}}$ is about two times the effective wire width $W_{\text {eff }} \approx 0.55 R_{\mathrm{c}}$. In our case $B_{\max }=0.37 \mathrm{~T}$, which corresponds to $R_{\mathrm{c}}=251 \mathrm{~nm}$ and $W_{\text {eff }} \approx 138 \mathrm{~nm}$. With a given lithographical width $W_{\text {lit }}=600 \mathrm{~nm}$ of the Hallbar, the depletion length from the sample edges can be estimated to be $W_{\text {dep }}=231 \mathrm{~nm}$.

The comparison of the suspended and nonsuspended quantum dot phonon cavity as shown in the insets of Fig. 5 leads to the conclusion that the quality of the electron gas is identical. The only alteration found is an enhanced boundary scattering introduced by the wet etching process, which is identified by the $B$-periodic oscillations in the low field region. We can conclude that investigation of the electronphonon interaction in cavities is within reach, as will be discussed elsewhere [11].

To summarize we have shown how to build and test qubits in coupled quantum dots. A single qubit is defined in two dots via tunnel splitting of the discrete states. This tunnel split state is traced in transport measurements and coherence is probed in an $\mathrm{AB}$ geometry. In further studies we have shown how to fabricate freely suspended electron gases in $\mathrm{AlGaAs} / \mathrm{GaAs}$-heterostructures. In a first step we characterized the electronic properties of the suspended electron-phonon cavities. We found that laterally gated quantum dots are the ideal testbed for the physics of quantum computation, i.e. phenomena considering phase coherence, dissipation and the fundamentals of the measuring process.

\section{Acknowledgements}

We acknowledge financial support by the Deutsche Forschungsgemeinschaft through the Schwerpunkt 'Quanteninformationsverarbeitung' (Bl/487-2-2), the Sonderforschungsbereich SFB-348 and by the Bundesministerium für Forschung und Technologie (BMBF-project 01BM/914 and the GermanIsraeli-Project DIP), and the Defense Advanced Research Projects Agency (DARPA-Ultra-Fast Electronics project). A.K.H. gratefully acknowledges support by the Studienstiftung des deutschen Volkes and the Stiftung Maximilianeum. H.Q. likes to thank the Volkswagenstiftung for support.

\section{References}

[1] L.P. Kouwenhoven, C.M. Marcus, P.L. McEuen, S. Tarucha, R.M. Westervelt, N.S. Wingreen, in: L.L. Sohn, L.P. Kouwenhoven, G. Schön (Eds.), Mesoscopic Electron Transport, Series E, Vol. 345, Kluwer Academic Publishers, Dordrecht, Netherlands, 1997, p. 105.

[2] A.K. Hüttel, H. Qin, A.W. Holleitner, R.H. Blick, K. Neumaier, D. Weinmann, K. Eberl, J.P. Kotthaus, 2002; cond-mat/0109014, submitted for publication.

[3] R.H. Blick, V. Gudmundsson, R.J. Haug, K. von Klitzing, K. Eberl, Phys. Rev. Rap. Comm. 57 (1998) R12 685.

[4] A.W. Holleitner, H. Qin, C.R. Decker, K. Eberl, R.H. Blick, Phys. Rev. Lett. 87 (2001) 256802.

[5] A.W. Holleitner, R.H. Blick, A.K. Hüttel, K. Eberl, J.P. Kotthaus, Science 297 (2002) 70.

[6] J. Kirschbaum, et al., Appl. Phys. Lett. 81 (2002) 280; E.M. Hoehberger, et al., Physica E 12 (2002) 487.

[7] H. Qin, A.W. Holleitner, K. Eberl, R.H. Blick, Phys. Rev. B 64 (2001) R241302.

[8] T. Fujisawa, T.H. Oosterkamp, W.G. van der Wiel, B.W. Broer, R. Aguado, S. Tarucha, L.P. Kouwenhoven, Science 282 (1998) 932.

[9] T. Brandes, B. Kramer, Phys. Rev. Lett. 83 (1999) 3021.

[10] R.H. Blick, F.G. Monzon, W. Wegscheider, M. Bichler, F. Stern, M.L. Roukes, Phys. Rev. B 62 (2000) 17103.

[11] E.M. Hoehberger, et al., 2002, in preparation. 